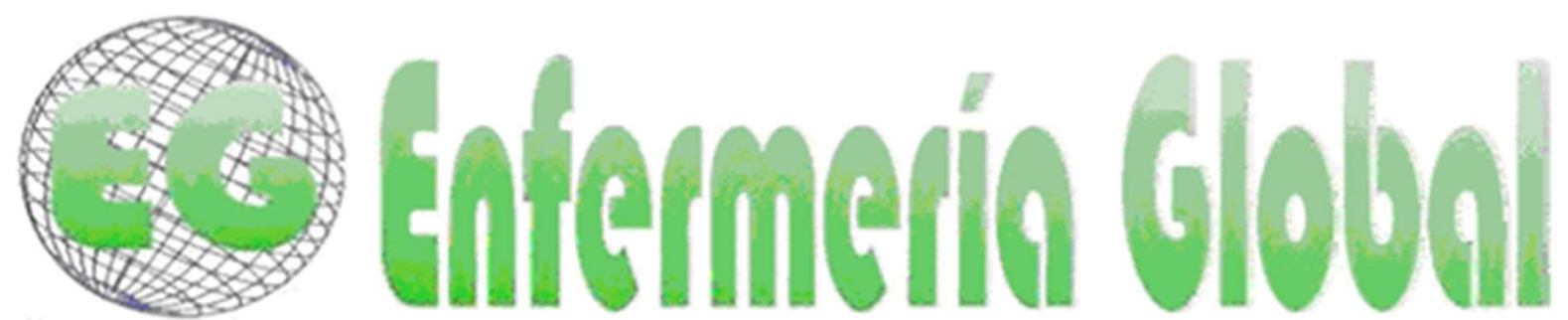

\title{
ORIGINALES
}

\section{Notificación de incidentes relacionados con la atención a la salud en un hospital docente}

Notificação de incidentes relacionados à assistência à saúde em um hospital de ensino

Notification of incidents related to health care in a teaching hospital

Valdenir Almeida da Silva ${ }^{1}$

Rosana Santos Mota ${ }^{1}$

Angela de Souza Barros ${ }^{1}$

Alessandra Rabelo Fernandes Gonçalves ${ }^{1}$

Monalisa Viana Sant'Anna ${ }^{1}$

Mara Regina Nascimento Barbosa dos Santos ${ }^{1}$

${ }^{1}$ Hospital Universitario Profesor Edgard Santos, Universidad Federal de Bahia. Salvador, Bahia, Brasil. valdenirenf@gmail.com

\section{https://doi.org/10.6018/eglobal.450481}

Recibido: $14 / 10 / 2020$

Aceptado: 3/03/2021

Financiación: Proyecto financiado con una beca de iniciación a la investigación por el programa Permanecer, edición 2019/2020, Pro-Rectoría de Acciones Afirmativas y Asistencia Estudiantil, Universidad Federal de Bahía.

\section{RESUMEN:}

Objetivo: Analizar los incidentes relacionados con la atención médica en un hospital docente.

Método: Investigación cuantitativa, realizada con base en las notificaciones de incidencias realizadas entre 2016 y 2018. Los datos se procesaron en la versión 12 del programa STATA.

Resultados: La incidencia de eventos adversos fue de 3,82 por cada 100 pacientes-día. Las unidades de hospitalización para adultos fueron los lugares con mayor incidencia de incidentes, 57,20\%; pacientes adultos, 52,75\%; mujeres, $52,9 \%$; negros, $80,01 \%$; solteros, $47,62 \%$; con escolarización baja o nula, el $50,91 \%$, fueron los principales. Las enfermeras fueron los principales notificadores, $80,38 \%$. Flebitis, 27,05\%; cirugías, 19,20\%; y las caídas, el 17,27\%, fueron los incidentes más reportados, cuyos daños fueron clasificados como leves en el 91,52\%, pero hubo 03 muertes en el período. Conclusión: El análisis de los incidentes permite destacar la importancia de las notificaciones para la planificación e implementación de medidas que puedan contribuir al fortalecimiento de la cultura de seguridad del paciente.

Palabras clave: Notificación; Eventos adversos; Seguridad del paciente; Hospitales de enseñanza; Enfermería 


\begin{abstract}
RESUMO:
Objetivo: Analisar os incidentes relacionados à assistência à saúde em um hospital de ensino. Método: Pesquisa quantitativa, realizada a partir das notificações de incidentes realizadas entre $2016 \mathrm{e}$ 2018. Os dados foram processados no programa STATA versão 12.

Resultados: A incidência de eventos adversos foi 3,82 por 100 pacientes-dia. As unidades de internação para adultos foram os locais com maior ocorrência de incidentes, 57,20\%; os pacientes adultos, $52,75 \%$; do sexo feminino, $52,9 \%$; negros, $80,01 \%$; solteiros, $47,62 \%$; com baixa ou nenhuma escolaridade, $50,91 \%$, foram os principais atingidos. Os enfermeiros foram os principais notificadores, $80,38 \%$. As flebites, $27,05 \%$; cirurgias, $19,20 \%$; e quedas, $17,27 \%$, foram os incidentes mais notificados, cujos danos foram classificados como leves em $91,52 \%$, mas houve 03 óbitos no período. Conclusão: A análise dos incidentes permite destacar a importância das notificações para o planejamento e implementação de medidas que possam contribuir para o fortalecimento da cultura de segurança do paciente.
\end{abstract}

Palavras-chave: Notificação; Eventos adversos; Segurança do paciente; Hospitais de ensino; Enfermagem.

\begin{abstract}
:
Objective: Analyzing incidents related to health care in a teaching hospital.

Method: A quantitative research carried out based on notifications of incidents carried out between 2016 and 2018. The data were processed in STATA version 12.

Results: The incidence of adverse events was 3.82 per 100 patient-days. The adult hospitalization units were the main notifiers, $57.20 \%$; adult patients, $52.75 \%$; females, $52.9 \%$; blacks, $80.01 \%$; singles, $47.62 \%$; with low or no schooling, $50.91 \%$, were the main ones. The nurses were the main notifiers, $80.38 \%$. Phlebitis, $27.05 \%$; surgeries, $19.20 \%$; and falls, $17.27 \%$, were the most reported incidents, whose damage was classified as mild in $91.52 \%$, but there were three deaths in the period. Conclusion: The analysis of incidents allows us to highlight the importance of notifications for the planning and implementation of measures that can contribute to the strengthening of the patient safety culture.
\end{abstract}

Key words: Notification, Adverse events, Patient safety, Teaching hospitals, Nursing

\title{
INTRODUCCIÓN
}

La seguridad de los pacientes es una preocupación mundial que afecta a todos los sistemas de salud tanto en los países desarrollados como en los países en desarrollo(1). En las organizaciones de salud, como organizaciones complejas cuyo riesgo nulo es imposible obtener, la existencia de mecanismos para gestionar incidentes relacionados con la atención de la salud y minimizar sus impactos contribuye al desarrollo de sistemas fiables ${ }^{(2)}$. Como estrategia para mejorar la atención y la asistencia prestada, los problemas relacionados con la seguridad del paciente han ido ganando protagonismo. A nivel mundial, la Organización Mundial de la Salud (OMS) puso en marcha, en 2004, la Alianza Mundial para la Seguridad del Paciente con el fin de fomentar los debates sobre el tema y estimular el desarrollo de acciones para la seguridad de los pacientes y la reducción de los eventos adversos $(\mathrm{EA})^{(3)}$.

El enfoque de la seguridad del paciente se ha enfatizado como una necesidad apremiante, según un editorial del periódico The Lancet "La seguridad del paciente no es un lujo" (Patient safety is not a luxury). Esta publicación refuerza la importancia de estructurar los sistemas y entornos de los servicios de salud capaces de promover la reducción de errores ${ }^{(4)}$. La OMS deduce que, en el mundo, hay un evento adverso evitable por cada 10 pacientes durante el uso de los servicios de salud(5). Se estima que 4,2 millones de pacientes en todo el mundo sufren daños o muerte anualmente debido a prácticas de atención inseguras o errores. El costo anual derivado de estas 
prácticas se estima en 42.000 millones de dólares, consumiendo alrededor del $1 \%$ de los gastos sanitarios mundiales ${ }^{(1)}$.

Dado que los riesgos de seguridad en el proceso de atención son inevitables, aunque en parte prevenibles ${ }^{(1,5)}$, se destaca la función de notificar incidentes relacionados con la atención sanitaria, cuyo objetivo principal es comunicar amenazas a la seguridad, como fallas cercanas, incidentes o eventos adversos, y la posibilidad de apoyar el aprendizaje de la experiencia. Un buen sistema de notificación interna se puede utilizar para identificar las amenazas a la seguridad del paciente y garantizar que todos los involucrados estén al tanto de tales amenazas. Por lo tanto, las notificaciones son importantes para monitorear los progresos en la prevención de errores, para permitir el monitoreo de prácticas seguras y como una manera de mejorar la seguridad del paciente ${ }^{(1,6-8)}$. Los sistemas de notificación permiten la recogida de información, análisis y difusión de las lecciones aprendidas ${ }^{(8)}$.

La información obtenida de las notificaciones es determinante para los responsables de la toma de decisiones, responsables del desarrollo y la gestión de las políticas de seguridad, los profesionales de la salud y los pacientes ${ }^{(9)}$, y puede apoyar la implementación de mejoras organizativas ${ }^{(6,10)}$. La toma de decisiones basadas en datos sobre notificaciones de incidentes, entonces, debe ser un compromiso asumido por los agentes involucrados en políticas públicas para la seguridad del paciente ${ }^{(2)}$.

Dada la importancia atribuida a las notificaciones de incidentes relacionados con la atención sanitaria para la seguridad del paciente, se decidió llevar a cabo este estudio, que tiene como objetivo analizar los incidentes relacionados con la atención sanitaria en un hospital docente.

\section{METODOLOGÍA}

Se trata de una investigación descriptiva y exploratoria con un enfoque cuantitativo, realizada a partir de datos secundarios de notificaciones de incidencias relacionadas con la atención sanitaria, refiriéndose a los años 2016, 2017 y 2018.

La investigación se llevó a cabo en un gran hospital docente (HD), perteneciente al Sistema Unificado de Salud (SUS), miembro de la red de la Empresa Brasileña de Servicios Hospitalarios, ubicada en Salvador, Bahía, Brasil. Es un hospital parte de la Red de Hospitales Centinelas de la Agencia Nacional de Vigilancia Sanitaria (ANVISA), cuyo objetivo principal es ser un observatorio activo del rendimiento y la seguridad de los productos utilizados en la salud. Como tal, colabora con la notificación de eventos adversos y quejas técnicas relacionadas con productos bajo vigilancia posterior al uso o posterior a la comercialización en el Sistema Nacional de Vigilancia Sanitaria (NOTIVISA). Además, cuenta con un Centro estructurado de Seguridad del Paciente con la implementación de los protocolos básicos de seguridad del paciente recomendados por ANVISA, además de protocolos de seguimiento e incidencias, y actividades de educación continua en seguridad del paciente.

El hospital cuenta con una aplicación de Vigilancia Hospitalaria (VIGIHOSP) implementada a mediados de 2015 para recibir notificaciones de incidentes y quejas técnicas relacionadas con la atención médica. Desde entonces, ha recibido 
notificaciones de todos los sectores del hospital siendo accesible a todos los profesionales.

Los datos fueron recogidos de agosto de 2019 a marzo de 2020 por un becario de iniciación a la investigación debidamente capacitado y supervisado por los investigadores responsables. Como VIGIHOSP se implementó en el hospital a mediados de 2015, se determinó como período de investigación los años 2016 a 2018, siendo este último el año completo antes de la preparación del proyecto.

Se incluyeron todos los informes de incidentes relacionados con la atención médica realizados en VIGIHOSP durante el período de investigación. Se recogieron variables relacionadas con los pacientes e incidentes, tales como: edad, raza, género, estado civil, nivel de educación, origen, turno de ocurrencia, categoría profesional del notificador, naturaleza de la notificación (anónima o identificada); fecha y lugar de ocurrencia; tipo y características del incidente; y grado de daño (leve, moderado, grave, muerte).

Se adoptó la clasificación del grado de daño según la Organización Mundial de la Salud (OMS)(11), en: 1) ninguno - no hubo ninguna consecuencia para el paciente; 2) leve - el paciente presentó síntomas leves, daño mínimo o intermediarios a corto plazo sin intervención o con una intervención mínima (pequeño tratamiento u observación); 3) moderado - el paciente requirió intervención, como la realización de un procedimiento adicional o terapia adicional, la prolongación de la hospitalización, la pérdida de la función, el daño permanente o a largo plazo; 4) grave - intervención necesaria para salvar la vida, intervención médico-quirúrgica importante o daños permanentes o a largo plazo importantes; perturbación/riesgo fetal o anomalía congénita; y 5) muerte. Cabe señalar que un incidente relacionado con la atención médica es un evento o circunstancia que podría resultar o que resultó en daños innecesarios al paciente. Cuando un incidente de este tipo resulta en daño al paciente, se llama un evento adverso(11).

Los datos se analizaron utilizando el software estadístico STATA versión 12 aplicando estadística descriptiva. La tasa de incidencia de incidentes relacionados con la atención sanitaria se calculó por la relación entre el número de incidentes notificados durante el período de estudio, dividido por el número total de pacientes ingresados en el mismo período $\times 100$.

El proyecto cumplió con los principios éticos de la investigación con seres humanos y cumplió con las recomendaciones de la Resolución 466/2012 del Consejo Nacional de Salud. El protocolo de investigación fue aprobado por un Comité de Ética en Investigación a través del número CAAE 09076619.2.0000.0049.

\section{RESULTADOS}

Durante el período de investigación, se reportaron 1911 incidentes en el HD, de los cuales 259 ocurrieron en 2016, 617 en 2017 y 1035 en 2018. La incidencia global de notificaciones durante el período de estudio fue de 7,94 por cada 100 pacientes hospitalizados-día, siendo 3,46 en 2016 ; 7,91 en 2017 y 11,78 en 2018. Las principales unidades notificantes fueron: unidades de hospitalización de adultos, con $1093(57,20 \%)$ notificaciones; sala de operaciones, con 240 (12,56\%) notificaciones; 
Unidad de Cuidados Intensivos para Adultos (UCI), con $203(10,62 \%)$ notificaciones; y la unidad de oncohematología, con 169 (8,84\%). (Tabla 1)

Tabla 1: Caracterización de los incidentes notificados relacionados con la atención sanitaria, según el lugar de ocurrencia. Salvador, Bahía, Brasil, 2020

\begin{tabular}{lcc}
\hline Lugar de ocurrencia & $\mathbf{n = 1 9 1 1}$ & $\%$ \\
\hline Unidades de hospitalización para & 1093 & 57,20 \\
adultos & 240 & 12,56 \\
Centro quirúrgico & 203 & 10,62 \\
UCl para adultos & 169 & 8,84 \\
Oncohematología & 115 & 6,02 \\
Unidades pediátricas & 13 & 0,68 \\
Ambulatorio de quimioterapia & 9 & 0,47 \\
UCl pediátrica & 7 & 0,37 \\
Unidad de Oftalmología & 7 & 0,37 \\
Ambulatorio & 3 & 0,16 \\
Hemodinámica & 3 & 0,16 \\
Boimage & 1 & 0,05 \\
Endoscopia & 1 & 0,05 \\
Hemodiálisis & 47 & 2,46 \\
Sin registro & & \\
\hline
\end{tabular}

La caracterización sociodemográfica de los pacientes con incidentes reportados apunta a personas mayoritariamente adultas, $1.008(52,75 \%)$ y ancianas, 649 (33,96\%); mujeres, 995 (52,9\%); autodeclaradas como negras, 1529 (80,01\%); solteras, 910 (47,62\%); con baja o ninguna escolaridad, $973(50,91 \%)$; y de Salvador - Bahía, 1030 (53,90\%). (Tabla 2)

Tabla 2: Caracterización sociodemográfica de pacientes con notificación de incidencias relacionadas con la asistencia sanitaria. Salvador Bahía, Brasil, 2020

\begin{tabular}{lcc}
\hline Variables sociodemográficas & $\mathbf{n = 1 9 1 1}$ & $\mathbf{\%}$ \\
\hline Edad & 111 & 5,81 \\
0 a 09 años & 92 & 4,81 \\
10 a 19 años & 1008 & 52,75 \\
20 a 59 años & 649 & 33,96 \\
60 años o más & 51 & 2,67 \\
Sin registro & & \\
Sexo & 995 & 52,09 \\
Mujer & 892 & 46,70 \\
Hombre & 23 & 1,20 \\
Sin registro & & \\
Raza & 1529 & 80,01 \\
Negra & 277 & 14,50 \\
No-negra & 105 & 5,49 \\
Sin registro & & \\
Estado civil & 910 & 47,62 \\
Soltero & 621 & 32,50 \\
Casado/unión estable & 80 & 4,19 \\
Separado & 129 & 6,75 \\
Viudo & &
\end{tabular}


Sin registro

Educación

Escuela primaria

Escuela secundaria

Enseñanza superior

Menor fuera de edad escolar

Ninguna

Sin registro

Origen

Salvador

Otro municipio

Sin registro

171

8,95

$\begin{array}{cc}818 & 42,80 \\ 541 & 28,31 \\ 113 & 5,91 \\ 33 & 1,73 \\ 155 & 8,11 \\ 251 & 13,13 \\ & \\ 1030 & 53,90 \\ 770 & 40,29 \\ 111 & 5,81\end{array}$

En cuanto a la caracterización de los incidentes notificados (Tabla 3), la mayoría se produjo durante la hospitalización del individuo en el HD, 1827 (95,70\%); en el turno matutino, 414 (21,66\%); con notificación identificada, 1446 (75,67\%); siendo el profesional de enfermería el principal notificador, $1536(80,38 \%)$. Se destaca la alta proporción de falta de registro en el turno de ocurrencia de los incidentes, 815 $(42,65 \%)$.

Tabla 3: Caracterización de los incidentes notificados relacionados con la salud. Salvador Bahía, Brasil, 2020

\begin{tabular}{lcc}
\hline Características de los incidentes & $\mathbf{n = 1 9 1 1}$ & \% \\
\hline Situación de la Ocurrencia & 1827 & 95,70 \\
Hospitalización & 32 & 1,68 \\
Sin registro & 26 & 1,36 \\
Atención ambulatoria & 24 & 1,26 \\
Otro & & \\
Turno de la Ocurrencia & 414 & 21,66 \\
Mañana & 353 & 18,47 \\
Tarde & 329 & 17,22 \\
Noche & 815 & 42,65 \\
Sin registro & & \\
Naturaleza de la Ocurrencia & 1446 & 75,67 \\
Identificada & 461 & 24,12 \\
Anónima & 04 & 0,21 \\
Sin registro & & \\
Profesional Notificador & 1536 & 80,38 \\
Enfermera & 163 & 8,53 \\
Farmacéutico & 88 & 4,60 \\
Técnico de enfermería & 67 & 3,51 \\
Médico & 27 & 1,41 \\
Estudiante & 18 & 0,94 \\
Nutricionista & 9 & 0,47 \\
Otros & 3 & 0,16 \\
Sin registro & & \\
\hline
\end{tabular}

En relación al tipo de incidente (Tabla 4), las mayores proporciones de notificaciones estaban relacionadas con la flebitis, 517 (27,05\%); cirugías, 367 (19,20\%); caídas, 330 (17,27\%); problemas relacionados con la cadena de medicamentos, 233 
$(12,19 \%)$; lesiones cutáneas, $181(9,47 \%) ;$ y problemas con la identificación del paciente, $72(3,77 \%)$.

Tabla 4: Tipos de los incidentes notificados relacionados con la salud. Salvador, Bahía, Brasil, 2020

\begin{tabular}{|c|c|c|}
\hline Incidente notificado & $n=1911$ & $\%$ \\
\hline Flebitis & 517 & 27,05 \\
\hline Cirugía & 367 & 19,20 \\
\hline Caídas & 330 & 17,27 \\
\hline Medicamentos & 233 & 12,19 \\
\hline Lesión de piel & 181 & 9,47 \\
\hline Identificación del paciente & 72 & 3,77 \\
\hline Artículo médico-hospitalario & 33 & 1,73 \\
\hline Terapia nutricional & 32 & 1,67 \\
\hline Pérdida de catéter & 24 & 1,26 \\
\hline $\begin{array}{l}\text { Sangre y/o componentes } \\
\text { sanguíneos }\end{array}$ & 8 & 0,42 \\
\hline Extubación accidental & 8 & 0,42 \\
\hline $\begin{array}{l}\text { Equipamientos médicos y } \\
\text { hospitalarios }\end{array}$ & 4 & 0,21 \\
\hline Error de diagnóstico & 2 & 0,10 \\
\hline Otros & 100 & 5,23 \\
\hline
\end{tabular}

En cuanto al daño, $920(48,14 \%)$ de los incidentes fueron clasificados como eventos adversos, con repercusiones en la salud de las personas. La incidencia de eventos adversos fue de 3,82 por cada 100 pacientes hospitalizados/día. Los incidentes que presentaron la mayor proporción de daños a la salud fueron: flebitis, lesiones cutáneas, artículos y equipamientos médico-hospitalarios, pérdida de catéter, sangre y/o componentes sanguíneos, extubación accidental y error diagnóstico, con daños a pacientes en el $100 \%$ de los casos, por lo que se clasificaron como eventos adversos; terapia nutricional, en $18(56,26 \%)$; caídas, en $43(13,03 \%)$; y problemas relacionados con la cadena de medicamentos, con $30(12,88 \%)$ casos.

Refiriéndose al grado de daño resultante del evento adverso (Tabla 5), la mayoría tuvo daños leves, 842 (91,52\%); 64 (6,96\%) causaron daños moderados; y tres $(0,32 \%)$ culminaron con la muerte del individuo. Los eventos que presentaron las mayores proporciones de daño considerados moderados o graves fueron: cirugía $(36,84 \%)$, equipo médico-hospitalario $(25 \%)$, medicamentos $(20 \%)$, extubación accidental $(12,50 \%)$, caídas $(11,63 \%)$ y lesiones cutáneas $(8,84 \%)$.

Tabla 5: Grado de daño de los eventos adversos notificados. Salvador Bahía Brasil, 2020

\begin{tabular}{lccccc}
\hline & \multicolumn{4}{c}{ Grado de daño N (\%) } \\
\hline Incidente & Leve & Moderado & Grave & Muerte & Sin registro \\
\hline Flebitis & $499(96,52)$ & $17(3,29)$ & - & - & $1(0,19)$ \\
Cirugía & $165(91,16)$ & $15(8,29)$ & $1(0,55)$ & - & - \\
Caídas & $38(88,37)$ & $5(11,63)$ & - & - & - \\
Medicamentos & $30(90,91)$ & $2(6,06)$ & - & - & $1(3,03)$ \\
Lesión de piel & $24(80,00)$ & $4(13,34)$ & $1(3,33)$ & $1(3,33)$ & -
\end{tabular}




\begin{tabular}{|c|c|c|c|c|c|}
\hline $\begin{array}{l}\text { Identificación del } \\
\text { paciente }\end{array}$ & $22(91,67)$ & $2(8,33)$ & - & - & - \\
\hline $\begin{array}{l}\text { Artículo médico- } \\
\text { hospitalario }\end{array}$ & $18(100,00)$ & - & - & - & - \\
\hline Terapia nutricional & $8(100,00)$ & - & - & - & - \\
\hline Pérdida de catéter & $7(87,50)$ & $1(12,50)$ & - & - & - \\
\hline $\begin{array}{ll}\text { Sangre } & \text { y/o } \\
\text { componentes } & \\
\text { sanguíneos } & \end{array}$ & $7(36,84)$ & $7(36,84)$ & - & - & $5(26,32)$ \\
\hline $\begin{array}{l}\text { Extubación } \\
\text { accidental }\end{array}$ & $3(100,00)$ & - & - & - & - \\
\hline $\begin{array}{l}\text { Equipamientos } \\
\text { médicos } \\
\text { hospitalarios }\end{array}$ & $2(50,00)$ & $1(25,00)$ & - & - & $1(25,00)$ \\
\hline Error de diagnóstico & $2(100,00)$ & - & - & - & - \\
\hline Otros & $17(56,67)$ & $10(33,34)$ & - & $2(6,66)$ & $1(3,33)$ \\
\hline TOTAL & $842(91,52)$ & $64(6,96)$ & $2(0,22)$ & $3(0,32)$ & $9(0,98)$ \\
\hline
\end{tabular}

\section{DISCUSIÓN}

Durante el período de estudio, la incidencia general de incidentes notificados relacionados con la atención médica fue de 7,94 por cada 100 pacientes-día. Entre los incidentes reportados, el $48,14 \%$ causó algunos daños a los pacientes y, por lo tanto, se clasificaron como acontecimientos adversos. La incidencia de acontecimientos adversos fue de 3,82 por cada 100 pacientes hospitalizados. Un estudio realizado en un hospital docente de Minas Gerais basado en notificaciones espontáneas en un sistema de notificación electrónica, durante un período de cuatro años, encontró un resultado similar, con una prevalencia de 33,8 incidentes por cada 1.000 hospitalizaciones ${ }^{(12)}$.

Estos datos difieren de otros estudios brasileños e internacionales sobre el porcentaje de eventos adversos entre los incidentes estudiados. Un estudio realizado en tres hospitales docentes en Río de Janeiro - RJ mostró una incidencia de eventos adversos del 7,6\%, con una proporción de 0,8 EA por cada 100 pacientes-día(13). Otro estudio identificó un porcentaje de $7,9 \%{ }^{(14)}$, y del $8,2 \%$, siendo que el $24 \%$ de los pacientes sufrieron entre dos y cinco eventos diferentes ${ }^{(15)}$ En otras publicaciones, todavía hay un mayor porcentaje de eventos adversos en unidades especializadas, como $\mathrm{UCl}$, cuya incidencia fue del $32,4 \%$, con una media de 2,8 eventos adversos por cada 100 pacientes-día ${ }^{(16)}$ y entre los ancianos hospitalizados, con una prevalencia del $58,8 \%$ y una media de 2,04 eventos por cada 100 hospitalizaciones ${ }^{(17)}$. A nivel internacional, cabe destacar que en Kenia se identificó una prevalencia del $1,4 \%$ de los eventos adversos, sobre la base de un examen de los registros médicos ${ }^{(18)}$; en Canadá, un estudio mostró un porcentaje de EA de $12,5 \%$ (19) y en Portugal, la incidencia de EA se estimó en $15,3 \%$ (20).

Es necesario reconocer que varios factores influyen en la ocurrencia de EA, como la cantidad y la calidad de los materiales y equipamientos disponibles, las condiciones estructurales del servicio y el acceso a las nuevas tecnologías ${ }^{(14)}$; el subdimensionamiento del personal de enfermería, la formación y la cualificación ${ }^{(14)}$, tiempo de experiencia profesional inferior a cinco años, para incidentes más leves y 
superior a cinco años para daños más graves ${ }^{(1)}$. La implementación de políticas de seguridad, por otro lado, favorece la identificación y notificación de incidentes, pasos necesarios para fortalecer la cultura de seguridad.

Un estudio realizado en un hospital universitario del sur de Brasil que tiene un dimensionamiento de personal adecuado identificó que la ocurrencia de eventos adversos fue mayor que en otros hospitales que también tienen un dimensionamiento dentro de la legislación recomendada, por lo tanto, es evidente que otros factores como la preparación inadecuada para procedimientos y diagnósticos quirúrgicos, el transporte inadecuado, la insuficiente formación del equipo también influyen en la ocurrencia de eventos adversos ${ }^{(14)}$.

Se observó que hubo un aumento en el número de notificaciones analizadas a lo largo de los años. En otros estudios, también se observó la misma tendencia de crecimiento con los años ${ }^{(1,6,7,12)}$ Se cree que este aumento se debe a la difusión de información sobre la aplicación utilizada para recibir notificaciones, la implementación de protocolos de seguridad del paciente y el desempeño de actividades educativas con los profesionales de la organización, cuando se destaca la importancia y estimulación de la práctica de notificación. La educación en servicio también es destacada por otros autores que encontraron un aumento en el número de notificaciones correlacionadas con entrenamientos mensuales ${ }^{(6)}$.

En el presente estudio, los principales orígenes de las notificaciones fueron las unidades de hospitalización de adultos $(57,20 \%)$; centro quirúrgico $(12,56 \%)$; UCI para adultos $(10,62 \%)$; y la unidad de oncohematología $(8,84 \%)$. Estos datos confirman una tendencia señalada en la literatura con respecto a los lugares con mayor ocurrencia de incidentes relacionados con la atención sanitaria(1,6,12,21). La mayor incidencia en las unidades hospitalarias puede estar relacionada con el mayor número de camas $\mathrm{y}$, en consecuencia, con el mayor número de pacientes hospitalizados. También se considera el perfil de gravedad, complejidad, inestabilidad hemodinámica, toma de una mayor cantidad de medicamentos y mayor sumisión a los procedimientos diagnósticos y terapéuticos de los pacientes ${ }^{(16)}$, como es el caso del centro quirúrgico, la $\mathrm{UCl}$ y la unidad de oncohematología como factores que predisponen los pacientes a sufrir incidentes de seguridad.

Las características de las personas que sufrieron algún incidente indican que el grupo de ancianos fue el segundo más afectado (33,96\%), por detrás de los adultos. También hubo un punto culminante para las mujeres; autodeclaradas negras, $80,01 \%$; solteras, 47,62\%; y con baja o ninguna escolaridad, 50,91\%. Otros estudios también muestran que los adultos y los ancianos conforman el grupo de edad que sufre más incidentes relacionados con la atención de la salud(1,12,15,16,22). También predominan las mujeres ${ }^{(14,15)}$ y sólo con la escuela primaria( ${ }^{(14)}$. El porcentaje de autodeclaración como perteneciente a la raza negra puede justificarse por el hecho de que alrededor del $80 \%$ de la población de la ciudad de Salvador - Bahía es afrodescendiente, la población más vulnerable y cuya mayoría utiliza los servicios de SUS, coincidiendo así con el origen de los pacientes hospitalizados que sufrieron incidentes.

Entre los incidentes reportados, el $95,7 \%$ se produjo durante la hospitalización, lo que puede justificarse por la mayor duración de la estancia del paciente en el servicio y, por lo tanto, estar más expuesto al riesgo. Cabe destacar que el $40,13 \%$ de los registros fueron incidencias que ocurrieron durante el día, principalmente por la 
mañana, y pueden explicarse por el mayor número de profesionales en los servicios, mayor vigilancia y mayor posibilidad de identificación y notificación de incidencias. En un estudio realizado en una UCl de un hospital en Sao Paulo - SP, el turno de la ocurrencia más reportada fue la noche $(36,8 \%)$ y hubo una estrecha distribución entre los turnos de mañana y de la tarde, pero el $77,4 \%$ de las notificaciones no informaron el turno de la ocurrencia, mientras que en esta investigación este porcentaje fue de $42,65 \%$ (12). Por la noche, hay menos profesionales de enfermería y del equipo multidisciplinario en el servicio, sin embargo, la distribución de incidentes en este turno fue menor. Sin embargo, se entiende que no siempre es posible identificar el turno de la ocurrencia del incidente, como es el caso de los acontecimientos que resultan de la evolución procesal, como la flebitis y la lesión por presión.

Las enfermeras fueron los mayores notificantes, representando el $80,38 \%$ del total de notificaciones en los tres años de la investigación. Estos datos son confirmados por otros autores ${ }^{(6,7)}$, sin embargo, en otras publicaciones los médicos aparecen como los mayores notificadores $^{(2)}$, además de los asistentes administrativos (Whitaker J, Ibrahim F, 2016), los técnicos de enfermería ${ }^{(6)}$ y los farmacéuticos ${ }^{(21)}$. Se sabe que los riesgos de incidentes deben ser compartidos entre el equipo multidisciplinario(23), así como la responsabilidad de las notificaciones.

Los incidentes con las proporciones más altas fueron flebitis, 27,05\%; relacionados con cirugías, 19,20\%; caídas, 17,27\%; problemas relacionados con la cadena de medicamentos, 12,19\%; lesiones cutáneas, 9,47\%; y problemas con la identificación del paciente, $3,77 \%$. A pesar de la relevancia para la seguridad del paciente y la alta proporción encontrada, este incidente no se encuentra entre las notificaciones más comunes en los servicios de salud(24). Este resultado lleva a la posibilidad de que pueda haber un subinforme de flebitis, ya que la terapia de infusión y la existencia de pacientes con factores de riesgo de flebitis, como los ancianos, forman parte de la vida diaria de los servicios de salud ${ }^{(25)}$. Aunque la flebitis se considera un evento adverso, todavía no es parte de los protocolos básicos de seguridad del paciente de ANVISA. Esto puede ser un factor que influye negativamente en su identificación y notificación, ya que la adopción de protocolos puede contribuir a la realización de una atención más segura debido al hecho de guiar las conductas y la prevención de fallos ${ }^{(26)}$.

Con respecto a los incidentes relacionados con las cirugías, parte de ellos se refieren a la no continuación del protocolo de cirugía segura, lo que indica que puede haber una maduración del equipo con respecto a las preocupaciones con la seguridad del paciente en el contexto quirúrgico, así contribuyendo a la cultura de seguridad.

Se observa que los incidentes más reportados, con la excepción de flebitis y cirugías, están de acuerdo con la literatura, sólo con algunas variaciones en términos porcentuales $(12,14,15,17,19-21,27,28)$. Un estudio realizado en 12 grandes hospitales de la región metropolitana de Salvador - Bahía reportó cómo la mayoría de los eventos adversos lesiones por presión (88,9\%), caídas $(77,8 \%)$, errores de medicación $(75 \%)$, eliminación no programada de drenajes y tubos $(42,9 \%)$ y fallas en la identificación del paciente $(33,3 \%)^{(24)}$. En Kenia, un estudio muestra que los eventos más reportados se asociaron con medicamentos, uso de sangre y equipamiento médico-hospitalario ${ }^{(18)}$. En Austria, una investigación indica que los incidentes más reportados se refieren a cirugías (45\%), identificación de pacientes $(12 \%)$, errores en el manejo de 
medicamentos (9\%), equipamientos médico-hospitalarios (10\%) y fallos de comunicación $(6 \%)^{(7)}$.

La mayoría de los incidentes $(91,52 \%)$ causaron daños leves; los eventos con daño moderado correspondieron al 6,96\%; los graves fueron del 0,22\%; y el 0,32\% llevó el paciente a la muerte. Otros estudios indican la ocurrencia del $79,6 \%$ de daño leve, con efectos temporales en los pacientes. Los incidentes relacionados con la cadena de medicamentos fueron más frecuentes, así como graves $(82 \%)^{(12)}$. Los datos sobre los informes de eventos adversos en Indonesia apuntan a la ocurrencia del $19,71 \%$ de las lesiones leves, el $2,19 \%$ de las lesiones permanentes y el $8,76 \%$ de las muertes $^{(29)}$. Un estudio basado en notificaciones en el sistema NOTIVISA entre 2014 y 2016 identificó que, de un total de 63.933 eventos adversos relacionados con la atención sanitaria, $4.179(0,6 \%)$ llevaron a la muerte. Según datos de la investigación, sólo un estado brasileño no recibió notificación de muerte en el período estudiado. Los incidentes se asociaron con infecciones relacionadas con la atención a la salud, la administración de medicamentos y líquidos intravenosos, el uso de sangre y productos sanguíneos, artículos y equipamientos médicos, y problemas estructurales y de instalaciones ${ }^{(30)}$.

La Organización Mundial de la Salud reconoce que la ocurrencia de eventos adversos es un fracaso en la seguridad del paciente y que alrededor del $60 \%$ de los incidentes ocurridos podrían evitarse. Las estrategias de seguridad del paciente deben considerarse medidas capaces de prevenir riesgos y reducir la posibilidad de daños debidos a la atención médica (11). Los acontecimientos adversos imponen consecuencias a los pacientes y a los servicios de salud, como el aumento de los costos hospitalarios con hospitalizaciones, una estancia más larga en cama, una necesidad adicional de procedimientos diagnósticos y terapéuticos, un mayor consumo de medicamentos, el absentismo en el trabajo y el riesgo de muerte prematura $^{(20)}$.

Este estudio presenta como limitación el hecho de que se realizó con datos de notificaciones voluntarias realizadas en un sistema electrónico. Como tal, no se puede descartar la posibilidad de subinformaciones y, por lo tanto, no se puede revelar el panel de incidentes en su totalidad. Otro factor limitante es la falta de informaciones sobre algunos elementos, como la ubicación y el turno de la ocurrencia, los datos sobre la caracterización de pacientes e incidentes, y el grado de daño. Sin embargo, se sabe que las notificaciones voluntarias están muy extendidas en todo el mundo, siendo uno de los métodos más útiles para generar cambios de comportamiento porque permite aprender con sus propios errores. Cabe destacar que la no utilización de notificaciones con fines punitivos y disciplinarios favorece la adhesión a su práctica, así como la inversión en actividades de educación permanente con el fin de difundir prácticas sanitarias seguras y necesarias para fortalecer la cultura de la seguridad del paciente ${ }^{(12)}$.

\section{CONCLUSIÓN}

El análisis de incidentes relacionados con la atención sanitaria notificados voluntariamente durante un período de tres años mostró una incidencia general de notificaciones de 7,94 por cada 100 pacientes-día, de los cuales el 48,14\% causó algún daño a los pacientes. La incidencia de eventos adversos fue de 3,82 por cada 
100 pacientes hospitalizados. La flebitis fue el incidente más reportado, seguida por el grupo de cirugías y caídas. En total, el 91,52\% de los acontecimientos causaron daños leves, pero hubo tres muertes en el período.

Los resultados encontrados permiten destacar la importancia de las notificaciones voluntarias de incidentes como uno de los factores que contribuyen al fortalecimiento de los programas y la cultura de seguridad. El conocimiento y la determinación de la magnitud de los incidentes y sus características relacionadas, así como el análisis del perfil de los pacientes afectados, pueden apoyar la planificación local y la implementación de diversas medidas, tales como acciones educativas. Estos pueden ayudar a los profesionales a entender el significado y la importancia de las notificaciones, y, en última instancia, favorecer la mejora de la calidad y la seguridad del paciente.

\section{REFERENCIAS}

1. Gao X, Yan S, Wu W, Zhang R, Lu Y, Xiao S. Implications from China patient safety incidents reporting system.Therapeutics and Clinical Risk Management. 2019;15:259-267. http://dx.doi.org/10.2147/TCRM.S190117

2. Fragata J, Sousa $P$, Santos RS. Organizações de saúde seguras e fiáveis/confiáveis. Cap. 1. In.: Sousa P, Mendes W (Org.). Segurança do paciente: criando organizações de saúde seguras. 2.ed (revista e ampliada). Rio de Janeiro, RJ: CDEAD, ENSP, Fiocruz, 2019:20-40. https://doi.org/10.7476/9788575416426

3. Alves SAC, Pereira AGD, Delduque MC. Segurança do Paciente: Aspectos comparativos entre Brasil e Portugal. In.: Santos AO, Lopes LT. Coletânea direito à saúde: boas práticas e diálogos institucionais. [Internet]. Brasília: CONASS, 2018: 168-182. [cited 2020 Sep 15]. Available from: http://www.resbr.net.br/coletaneadireito-a-saude-conass/\#.XyqPzChKi73

4. Patient safety is not a luxury. The Lancet. 2016; 387(19):1133 doi: https://doi.org/10.1016/S0140-6736(16)30003-4

5. World Health Organization (WHO).World Alliance for Patient Safety. WHO patient safety curriculum guide: multi-professional edition [Internet]. Geneva: WHO; 2011 [cited 2016 Oct 31]. Available from: http://apps.who.int/iris/bitstream/10665/44641/1/9789241501958 eng.pdf.

6. Ramírez E, Martín A, Villán Y, Lorente M, Ojeda J, Moro M, et al. Effectiveness and limitations of an incident reporting system analyzed by local clinical safety leaders in a tertiary hospital. Prospective evaluation through real-time observations of patient safety incidents. Medicine. 2018; 97:38(e12509). http://dx.doi.org/10.1097/MD.0000000000012509

7. Sendlhofer G, Schweppe P, Sprincnik U, Gombotz V, Leitgeb K, Tiefenbacher $P$, et al. Deployment of critical Incident reporting system (CIRS) in public Styrian hospitals: a five year perspective. BMC Health Services Research. 2019;19:412. https://doi.org/10.1186/s12913-019-4265-0

8. Dhamanti I, Leggat S, Barraclough S, Liao HH, Abu Bakar N. Comparison of patient safety incident reporting systems in Taiwan, Malaysia, and Indonesia. Journal of Patient Safety. 2020; Volume Publish Ahead of Print. doi: 10.1097/PTS.0000000000000622

9. Golder S, Loke YK, Wright K, Norman G. Reporting of adverse events in published and unpublished studies of health care interventions: a systematic review. PLoS Med. 2016; 13(9): e1002127. doi:10.1371/journal.pmed.1002127 
10. Whitaker J, Ibrahim F. Incident reporting feedback experience in a UK secondary care setting. Are staff increasingly reluctant to complete incidence forms? The Bulletin. 2016; 98(2):82-84. doi: 10.1308/ rcsbull. 2016.82

11. World Health Organization (WHO). Conceptual Framework for the International Classification for Patient Safety [Internet]. Patient Safety: WHO Press; 2009. [cited 2020 Sep 15]. Available from: https://www.who.int/patientsafety/implementation/taxonomy/icps_technical_report_en .pdf

12. Figueiredo ML, Oliveira e Silva CS, Brito MFSF, D'Innocenzo M. Analysis of incidents notified in a general hospital. Rev Bras Enferm [Internet]. 2018;71(1):111-9. DOI: http://dx.doi.org/10.1590/0034-7167-2016-0574

13. Mendes W, Martins M, Rozenfeld S, Travassos C. The assessment of adverse events in hospital in Brazil. Int J Qual Health Care [Internet]. 2009 [cited 2017 Out 27]; 21(4): 279-84. Available from: https://www.aeciherj.org.br/publicacoes/eventoadverso-Brasil-2009.pdf

14. Sell BT, Amante LN, Martins T, Sell CT, Senna CVA, Loccioni MFL. Dimensionamento dos profissionais de enfermagem e a ocorrência de eventos adversos em internação cirúrgica. Cienc Cuid Saude. 2018; 17(1). doi: 10.4025/cienccuidsaude.v17i1.33213

15. Ortega DB, D'Innocenzo M, Silva LM, Bohomol E. Análise de eventos adversos em pacientes internados em unidade de terapia intensiva. Acta Paul Enferm. 2017; 30(2):168-73. http://dx.doi.org/10.1590/1982-0194201700026

16. Roque KE, Tonini T, Melo ECP. Adverse events in the intensive care unit: impact on mortality and length of stay in a prospective study. Cad. Saúde Pública. 2016; 32(10):e00081815. http://dx.doi.org/10.1590/0102-311X00081815

17. Teixeira CC, Bezerra ALQ, Paranaguá TTB, Pagotto V. Prevalência de eventos adversos entre idosos internados em unidade de clínica cirúrgica. Rev baiana enferm. 2017; 31(3):e22079. doi: 10.18471/rbe.v31i3.22079

18. Macharia WM, Muteshi CM, Wanyonyi SZ, Mukaindo AM, Ismail A, Ekea H, Abdallah A, Tole JM, Ngugi AK. Comparison of the prevalence and characteristics of inpatient adverse events using medical records review and incident reporting. S Afr Med J. 2016;106(10):1021-1036. doi:10.7196/SAMJ.2016.v106i10.10619

19. D'Amour D, Dubois CA, Tchouaket E, Clarke S, Blais R. The occurrence of adverse events potentially attributable to nursing care in medical units: cross sectional record review. Int J Nurs Stud. 2014; 51(6):882-91. http://dx.doi.org/10.1016/j. ijnurstu.2013.10.017

20. Sousa P, Uva AS, Serranheira F, Uva MS, Nunes C. Patient and hospital characteristics that influence incidence of adverse events in acute public hospitals in Portugal: a retrospective cohort study. Int J Qual Health Care. 2018; 30(2):132-7. http://dx.doi.org/10.1093/intghc/mzx190

21. Moreira IA. Notificação de eventos adversos: o saber e o fazer de enfermeiros. Dissertação (mestrado). Universidade Federal de Goiás. [Internet].Goiânia, 2018. [cited 2020 Sep 15]. Available from: http://repositorio.bc.ufg.br/tede/handle/tede/8759

22. Araújo ACQ, Silva VA, Mota RS, Mendes AS, Barros AS, Sant'Anna MV, et al. Incidentes relacionados à assistência à saúde em idosos hospitalizados. Rev enferm UFPE online. 2020;14:e244639. https://doi.org/10.5205/1981-8963.2020.244639

23. Amaral RT, Bezerra ALQ, Teixeira CC, Paranaguá TTB, Afonso TC, Souza ACS. Riscos e ocorrências de eventos adversos na percepção de enfermeiros assistenciais. Rev Rene. 2019;20:e41302. doi: 10.15253/2175-6783.20192041302 
24. Costa EAM, Lobão WM, Ribas CLM, Passos NM. Segurança do paciente em serviços de saúde: uma análise na cidade de Salvador, Bahia. Rev. SOBECC. 2020; 25(1):17-24. doi: 10.5327/Z1414-4425202000010004

25. Mota RS, Silva VA, Mendes AS, Barros AS, Santos OMB, Gomes BP. Incidência e caracterização das flebites notificadas eletronicamente em um hospital de ensino. Rev baiana enferm. 2020;34:e35971. doi:10.18471/rbe.v34.35971

26. Aozane F, Cigana DJ, Benetti ERR, Herr GEG, Kolankiewicz ACB, Pizolotto MF. Percepções de enfermeiros de um hospital privado sobre eventos adversos na assistência de enfermagem. Revenferm UFPE online. 2016;10(2):379-86. doi: 10.5205/reuol.8557-74661-1-SM1002201601

27. Costa NN, Silva AEBC, Lima JC, Barbosa MRS, Freitas JS, Bezerra ALQ. O retrato dos eventos adversos em uma clínica médica: análise de uma década. Cogitare Enferm [Internet]. 2016; 21(esp): 01-10. [cited 2020 Sep 15]; Available from: https://revistas.ufpr.br/cogitare/article/view/45661/pdf_1

28. Vossoughi S, Perez G, Whitaker BI, Fung MK, Rajbhandary S, Crews N, Stotler B. Safety incident reports associated with blood transfusions. Transfusion. 2019;59;2827-2832. doi: 10.1111/trf.15429

29. Komite Keselamatan Pasien Rumah Sakit. Pedoman Pelaporan Insiden Keselamatan Pasien (IKP). [Internet]. Jakarta: KKPRS; 2015. [cited 2020 Sep 15]. Available from: http://rspmanguharjo.jatimprov.go.id/wp-content/uploads/2020/02/1.PEDOMAN-PELAPORAN-INSIDEN-KESELAMATAN-PASIEN-IKP-KKPRS-2008-

\section{EDISI-2.pdf}

30. Maia CS, Freitas DRC, Gallo LG, Araújo WN. Registry of adverse events related to health care that results in deaths in Brazil, 2014-2016. Epidemiol. Serv. Saude. 2018;27(2):e2017320. doi: 10.5123/S1679-49742018000200004 